\title{
Fast Beam Alignment for Millimeter Wave Time-Varying Channels Using Sparse Codes
}

\author{
Long Cheng, Guangrong Yue, Member, IEEE, Pei Xiao, Senior Member, IEEE, Ning Wei, and Shaoqian \\ Li, Fellow, IEEE,
}

\begin{abstract}
In this paper, a novel beam alignment algorithm based on the sparse graph coding theory is proposed for millimeter wave (mmWave) time-varying channels. Firstly, a pilot design method is introduced to transform the mmWave timevarying beam alignment into a sparse-graph design and detection problem. Inspired by Low-Density-Parity-Check (LDPC) codes and fountain codes, a multi-stage sparse coding method is proposed for the design of the measurement matrix and the theoretical bound of the probability of success is derived to guide the design of the sparse-graph. A beam alignment algorithm is subsequently proposed to detect the beam index and estimate the carrier frequency offset (CFO). Then, the Carmer-Rao Lower Bound (CRLB) is derived. Simulation results demonstrate that the proposed beam alignment algorithm achieves significant performance improvements over the conventional counterparts in both the noiseless and noise cases.
\end{abstract}

Index Terms-Beam alignment, time-varying channels, sparsegraph codes, Massive MIMO.

\section{INTRODUCTION}

Millimeter wave(mmWave) communication is considered as a key technology of the fifth-generation (5G) networks to dramatically scale up the system capacity by exploiting the abundant frequency spectrum $(30-300 \mathrm{GHz})[1]$. However, due to the the higher propagation loss compared with the lower frequency bands [1], large antenna arrays are required to provide sufficient beamforming gain. This brings difficulties to obtain the information of mmWave channels.

Recently, by leveraging the sparse nature of mmWave channels, some compressed sensing (CS)-based channel estimation algorithms are proposed [2], [3]. Nevertheless, those schemes always have a high computational complexity due to the largedimension matrix operations. To reduce the complexity, a class of beam scanning and searching schemes have been extensively studied, e.g., [4]-[7]. However, those schemes require either searching for all candidate beams [4], feedback links

This work was partly supported by the National Key Research and Development Program of China (No. 2020YFB1805001), in part by the National Natural Science Foundation of China (No. 61831004), in part by ZTE Corporation, and in part by the China Scholarship Council. The work of Pei Xiao was supported by the U.K. Engineering and Physical Sciences Research Council under Grant EP/P03456X/1. (Corresponding author: Guangrong Yue)

L. Cheng, G. Yue, and S. Li are with the National Key Laboratory of Science and Technology on Communications, University of Electronic Science and Technology of China, Chengdu 611731, China. (e-mail: chenglong_office@163.com, yuegr@uestc.edu.cn )

P. Xiao is with the 5GIC \&6GIC, Institute for Communication Systems (ICS), University of Surrey, United Kingdom

N. Wei is with the ZTE Corporation, Shenzhen 518057, China and also with the State Key Laboratory of Mobile Network and Mobile Multimedia Technology, Shenzhen 518055, China.
[5], or sending long PN sequences [6]. More importantly, most of the existing beam alignment (BA) algorithms [4]-[7] only consider the static channel model or low mobility scenarios, while the time-varying channels need to be considered for practical mmWave applications. In [7], a sparse coding based beam alignment algorithm is proposed for mmWave beam alignment in time-varying channels. However, in mobility scenario, it is necessary to obtain the Doppler shift of each path to compensate its effect rather than simple beam alignment. Furthermore, the algorithm only obtains the amplitude information, and the encoding construction and decoding algorithm are based on idealized assumption, the performance degrades significantly with short pilot sequence length.

Against the above background, the contributes of this paper can be summarized as follows:

1) A beam alignment algorithm framework based on sparsegraph coding and decoding is proposed. Firstly, we divide the measurement matrix into two parts, i.e., the sparse coding matrix and the detection matrix. Then, inspired by the erasure-correcting codes, e.g., fountain codes [8] and Low-Density-Parity Check (LDPC) codes [9], which both have low computational complexity, a similar coding matrix is designed. Furthermore, by utilizing the density evolution analysis method [10], we derive the theoretical bound as a benchmark to chose the optimal parameter of the designed coding matrix. In addition, the minimum probability of success of our algorithm is analytically determined by this theory bound, while the previous algorithm [7] can only determine a loose upper bound.Consequently, the minimum pilot overhead can be achieved by our proposed algorithm and which outperforms the similar algorithm [7], although the latter is based on the sparse coding.

2) A time-varying channel beam alignment algorithm is proposed to detect the defined single-ton and estimate the carrier frequency offset (CFO). Compared with the existing iterative sparse message passing algorithm [3], our proposed algorithm has a lower computational complexity. Furthermore, by modeling a successful bin detection as a three dimensional tensor, we derive the theoretical Carmér-Rao Lower Bound (CRLB) of the time-varying channel parameters.

Finally, simulation results show that the proposed sparse coding based algorithm outperforms the existing beam alignment algorithms [6], [7]. Furthermore, in some cases, our algorithm approaches the CRLB for both the success rate and 
the CFO estimation.

\section{SySTEM MODEL}

This paper considers a typical downlink mmWave massive MIMO system, where the BS is equipped with $N_{\mathrm{T}}$ antennas and $N_{\mathrm{RF}}$ radio frequency $(\mathrm{RF})$ chains, and the mobile station (MS) utilizes $M_{\mathrm{R}}$ antennas and $M_{\mathrm{RF}}$ radio RF chains. To incorporate the oscillator frequency offset (OFO) and the Doppler frequency offset (DFO) with conventional mmWave channels, the mmWave time-varying channel can be expressed as [11]

$$
\mathbf{H}(t)=\sum_{l=1}^{L} a_{l} \boldsymbol{\alpha}_{\mathrm{MS}}\left(\theta_{l}\right) \boldsymbol{\alpha}_{\mathrm{BS}}^{H}\left(\varphi_{l}\right) e^{j 2 \pi f_{l}^{c} T_{s} t},
$$

where $\left\{a_{l}, \theta_{l}, \varphi_{l}\right\}$ denote the complex gain, the AoA, and the AoD of the $l$-th path, respectively. In addition, $f_{l}^{c}=f_{l}^{d}+\varepsilon$ is the CFO for the $l$-th path, where $f_{l}^{d}$ denotes the DFO and $\varepsilon$ is the OFO between the transceiver and the receiver. And $T_{s}$ is the sampling period. Furthermore, without loss of generality, we consider utilizing the uniform linear array (ULA) at the BS and the UE, and $\boldsymbol{\alpha}_{\mathrm{BS}}($.$) and \boldsymbol{\alpha}_{\mathrm{MS}}($.$) denote the array response$ vectors.

Different from the traditional beam alignment algorithms, which only emit a single beam at a time, in our pilot design, the BS probes the channel with multiple beams and transmits constant signal in one training period. The transmitted beamforming vector can thus be written as

$$
\mathbf{f}(t)=\mathbf{F}_{\mathrm{BS}} \boldsymbol{\psi}(t),
$$

where $\mathbf{F}_{\mathrm{BS}} \in \mathbb{C}^{N_{\mathrm{T}} \times N_{\mathrm{T}}}$ denotes the Discrete Fourier Transform (DFT) matrix, and $\psi(t) \in \mathbb{C}^{N_{\mathrm{T}} \times 1}$ is the beamcoding vector. Similarly, the UE can locally customize its own beamforming codebook, such as $\mathbf{w}(t)=\mathbf{F}_{\mathrm{MS}} \mathbf{v}(t) \in \mathbb{C}^{M_{\mathrm{R}} \times M_{\mathrm{RF}}}$. Specially, the UE utilizes $M_{\mathbf{U E}} \leq M_{\mathbf{R F}}$ beams to exhaust the whole beam index. Thus, after the standard time synchronization and carrier recovery, the received signal can be expressed as

$$
\begin{aligned}
\mathbf{r}(t) & =\mathbf{w}^{H}(t) \mathbf{H}(t) \mathbf{f}(t)+\mathbf{n}(t) \\
& =\mathbf{v}^{H}(t) \mathbf{F}_{\mathrm{MS}}^{H} \mathbf{F}_{\mathrm{MS}} \widehat{\mathbf{H}}(t) \mathbf{F}_{\mathrm{BS}}^{H} \mathbf{F}_{\mathrm{BS}} \psi(t)+\mathbf{n}(t) \\
& =\mathbf{v}^{H}(t) \mathbf{I}_{\mathrm{MS}} \widehat{\mathbf{H}}(t) \mathbf{I}_{\mathrm{BS}} \psi(t)+\mathbf{n}(t) \\
& =\mathbf{v}^{H}(t) \widehat{\mathbf{H}}(t) \psi(t)+\mathbf{n}(t)
\end{aligned}
$$

where $\widehat{\mathbf{H}}(t) \in \mathbb{C}^{M_{\mathrm{R}} \times N_{\mathrm{T}}}$ denotes the virtual angle domain representation, $\mathbf{F}_{\mathrm{MS}}^{H} \mathbf{F}_{\mathrm{MS}}=\mathbf{I}_{\mathrm{MS}} \in \mathbb{C}^{M_{\mathrm{R}} \times M_{\mathrm{R}}}$ and $\mathbf{F}_{\mathrm{BS}}^{H} \mathbf{F}_{\mathrm{BS}}=$ $\mathbf{I}_{\mathrm{BS}} \in \mathbb{C}^{N_{\mathrm{T}} \times N_{\mathrm{T}}}$ denote the identity matrices, $\mathbf{n}(t) \in \mathbb{C}^{N_{\mathrm{RF}} \times 1}$ is the stacked noise vector with i.i.d $\mathcal{C N}\left(0, \sigma_{n}^{2}\right)$ entries.

Suppose the UE can obtain $N_{\mathrm{RF}}$ projections per time slot, thus, by utilizing a suitable signal splitter, the received signal of the $j$-th RF chain can be given by

$$
\mathbf{r}_{j}(t)=\widehat{\mathbf{h}_{\mathbf{s}}}(t) \boldsymbol{\psi}(t)+\mathbf{n}_{j}(t),
$$

where $\widehat{\mathbf{h}_{\mathbf{S}}}(t)$ denotes the $s$-th row of the virtual channel $\widehat{\mathbf{H}}(t)$. Since data from each RF link can be received separately and processed separately, then we only consider the data from a single RF chain. Here, we simplify each row of the beam channel as $\widehat{\mathbf{h}_{s}}(t)=\widehat{\mathbf{h}}(t)$. Furthermore, by collecting $T$

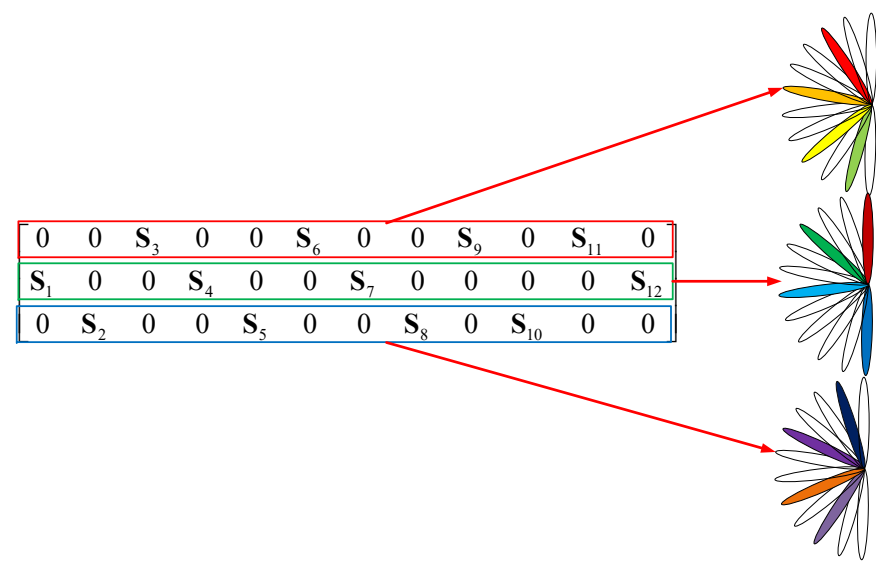

Fig. 1: A conceptual diagram of the sparse beamcoding matrix $\psi$ and the emitted beam, where different color denotes different bin detection beam.

received pilot signals and taking into account the time-varying channel, we obtain

$$
\mathbf{r}=\left[\begin{array}{c}
\boldsymbol{\psi}^{T}(1) \times \widehat{\mathbf{h}}(1) \\
\boldsymbol{\psi}^{T}(2) \times \widehat{\mathbf{h}}(2) \\
\vdots \\
\boldsymbol{\psi}^{T}(T) \times \widehat{\mathbf{h}}(T)
\end{array}\right]+\mathbf{n}
$$

where $\widehat{\mathbf{h}}(t)=\operatorname{vec}(\widehat{\mathbf{h}}(t)) \in \mathbb{C}^{M_{\mathrm{T}} \times 1}$ denotes the vectorized virtual angular domain representation, and $\mathbf{n}$ is the noise. Since the follow analysis is based on the single RF chain, we define $\mathbf{r}_{j}=\mathbf{r}$ and $\mathbf{n}=\mathbf{n}_{j}$ for simplicity whenever no ambiguity arises. In the sequel, we will discuss how to effectively design the beamcoding matrix $\psi \in \mathbb{C}^{T \times N_{\mathrm{T}}}$ and extract the beam index $\widehat{\mathbf{h}}(t)(t=1, . ., T)$ from the received signal without the index feedback mechanism. Due to the sparsity of millimeter-wave channels, we only need to obtain a few channel parameters, channel support $|\widehat{h}(t)|(t=1 \ldots T)$, complex gain $a_{l}$, and Doppler frequency shift $f_{l}^{d}$ of each path $l$.

\section{Proposed Beam Alignment Scheme}

\section{A. Proposed Algorithm Framework Based on Sparse Coding and Decoding}

In our scheme, the sparse beamcoding matrix $\boldsymbol{\psi} \in \mathbb{C}^{N M \times N_{\mathrm{T}}}$ is divided into two parts, the sparse coding matrix $\mathbf{G} \in \mathbb{C}^{M \times N_{\mathrm{T}}}$ and the bin detection matrix $\mathbf{S} \in \mathbb{C}^{N \times N_{T}}$. The measurement matrix $\psi$ can thus be given by

$$
\boldsymbol{\psi}=\mathbf{G} \odot \mathbf{S}
$$

where $\odot$ denotes the row-tensor operator. Mathematically, we rewrite the measurement matrix $\boldsymbol{\psi}$ as follows:

$$
\boldsymbol{\psi}=\left[\mathbf{G}_{1} \otimes \mathbf{S}_{1} \cdots \mathbf{G}_{M_{\mathrm{T}}} \otimes \mathbf{S}_{M_{\mathrm{T}}}\right],
$$

where $\mathbf{G}_{i}$ and $\mathbf{S}_{i}$ represent the $i$ column of the matrix $\mathbf{G}$ and the matrix $\mathbf{S}$, respectively, and $\otimes$ denotes the Kronecker product. Our design philosophy is depicted in Fig. 1 as a cartoon illustration, where the non-zero positions in the matrix represent different beam angles and different colors represent 

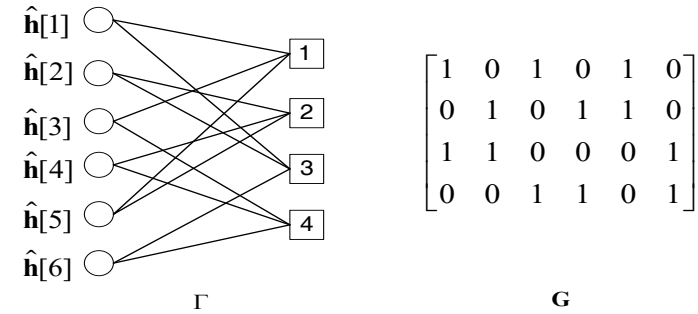

Fig. 2: An illustration of the regular bipartite graph with its associated sparse codes, while the left degree $b=2$.

different beam identification vectors. Next, we will discuss how to design these two matrices separately.

Since these two parts target at different functions of the decoding algorithm, the sparse coding matrix $\mathbf{G}$ can be designed independently from the bin matrix $\mathbf{S}$. Inspired by the traditional LDPC coding method [9], we construct a regular bipartite graph $\Gamma^{M_{T}}(R, b)$ with $N_{\mathrm{T}}$ left nodes and $R$ right nodes for the sparse coding matrix, where each left nodes is connected to $b$ right nodes at random [10], as shown in Fig. 2. Specifically, the design of the coding matrix $G$ follows the "beams-and-bins" philosophy, where each non-zero entry $\left(\mathbf{G}_{i, j}=1\right)$ denotes that the $j$-th bin sends the $i$-th preset beam. Then, according to the distribution of multipath direction of mmWave channels, we can classify the observation detections based on its edge degree as follows:

1) zero-ton: A right node is a zero-ton if it is not connected any non-zero entry of the angle domain channel $\widehat{\mathbf{h}}$.

2) single-ton: A right node is a single-ton if it is connected to only one non-zero entry of the angle domain channel $\widehat{\mathbf{h}}$.

3) multi-ton: A right node is a multi-ton if it is connected to more than one non-zero entry of the angle domain channel $\widehat{\mathbf{h}}$.

To be specific, these three types represent the cases that the transmitting beams are aligned with zero path, one path, and multiple paths in the mmWave time-varying channel, respectively. In particular, it is unreasonable to assume that the multi-ton node will not appears, which is different from the conclusion in [7]. Then, we propose a more general decoding method compared with the phaseless decoding method [9]. Specially, if all the types of the node can be determine, similar to the message passing algorithm [9], a peeling decoder can be applied to peel off all the single-ton in the bipartite graph and the parameters of the channel can be obtained from the single-ton.

Finally, we carry out a probability analysis of the proposed peeling-decoder, over a graph which is randomly chosen from the regular graph ensemble $\Re^{K}(F, m)$. In this ensemble, the $m$-th detection is divided into $d$ stages, with each left node connected to one right node per stage randomly. The set $F$ is defined as $F=\left\{f_{1} \cdots f_{d}\right\}$, where the number of right nodes at stage $i$ is $f_{i}$. In particular, $f_{i}=\mu K+O(1)$. Then, we can design the coding matrix $\mathbf{G}$ so that each stage has a circularlyshifted subsampling pattern.

In the previous algorithm [7], the authors only deduce the
TABLE I: The Minimum Threshold Value of $\eta$ of the Number of Stage $d$

\begin{tabular}{c|c|c|c|c|c|c}
\hline $\mathrm{d}$ & 3 & 4 & 5 & 6 & 7 & 8 \\
\hline$\mu$ & 0.407 & 0.323 & 0.285 & 0.261 & 0.245 & 0.233 \\
\hline$d \mu$ & 1.221 & 1.292 & 1.425 & 1.566 & 1.715 & 1.864 \\
\hline
\end{tabular}

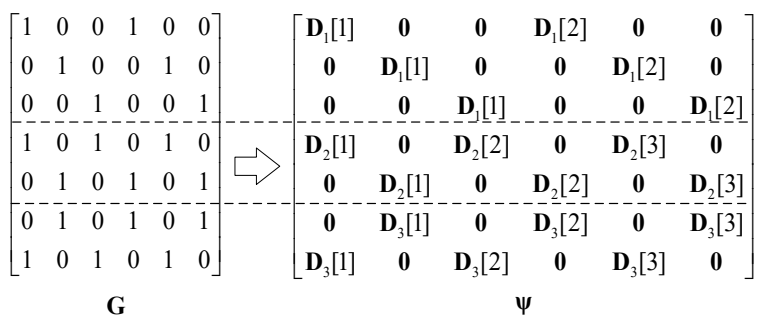

Fig. 3: An illustration of the sparse codes with its associated measurement matrix. Note that $\mathbf{D}_{i}[x]$ denotes the $x$-th column of the bin matrix $\mathbf{D}_{i}$.

upper bound of the beam alignemtn algorithm based on sparse coding.By utilizing the Doob martingale in [9], we show that the $2 l$-depth neighborhood of the edges of our proposed ensemble $\Re^{K}(F, m)$ is cycle-free. Then, using the density evolution analysis in [10], our proposed peeling decoder can recover $K$ sparse $\widehat{\mathbf{h}}$ with a probability given as follows:

Theorem 1: If the proposed peeling decoder over a random graph from the ensemble $\Re^{K}(F, m)$, satisfies the stages of $d \geq$ 3 and $f_{i}=\mu K+O(1)$ with the constant $\mu$ being chosen from Table $I$, then it can successfully recover $K$ non-zero entries with probability $1-O(1 / m)$.

Proof: See Appendix A.

\section{B. Proposed Beam Alignment Algorithm Using Sparse-Graph Codes}

In this subsection, a beam alignment algorithm using sparsegraph codes is proposed. Firstly, we can utilize the same regular graph $\Gamma^{M_{T}}(R, b)$ as mentioned in Section III-A to ensure high success beam detection probability. Then, we propose a new bin detection matrix $\mathbf{D}$ which contains part of the previous bin detection matrix $\mathbf{S}$.

Since only a few beams are transmitted at each time, we can also design the bin detection matrix $\mathbf{D}$ to be sparse, i.e., the beams in different directions have different weights each time. In particular, an example is shown in Fig. 3, each stage have $M_{i}$ modulation vector $\mathbf{D}_{i} \in \mathbb{C}^{P \times 1}$. Furthermore, in the presence of noise, each entry of the matrix $\mathbf{D}_{i}$ is randomly selected from a i.i.d Gaussian entries $\mathcal{N}(0,1)$.

Next, we propose a detection scheme for each bin. Consider the phase rotation introduced by the CFO, the mmWave channel gain varies in time. In the meantime, the magnitude of single path remains constant. Thus, we can utilize the magnitude information to detect the type of each bin. Firstly, we simple check if the received vector is zero-ton as follows:

$$
\left|\mathbf{r}_{i, j}[1]\right|^{2} \leq\left(\delta_{\min }^{2} \varepsilon_{1}+\sigma_{\min }^{2}\right),
$$

where $\delta_{\min }^{2}$ denotes the minimum signal power, $\sigma_{\min }^{2}$ is the minimum noise power, and $\varepsilon_{1}$ is the zero-ton detection 
threshold. Consequently, the right-hand side of the equation represents the smallest signal power associated with the noise. If the equation holds, then it is proved that the received data is less than all known signal powers.

We then assume that the received bin vector is a single-ton and then estimate the index pair $\langle\widetilde{b}, \widetilde{\mathbf{h}}[\widetilde{b}]\rangle$. Specifically, for bin $\mathrm{j}$ of stage $\mathrm{i}$, a maximum likelihood (ML) method is utilized to obtain the possible absolute value of the coefficient for the $k$-th column of the bin matrix $\mathbf{D}_{i}$ as follows:

$$
\left|\alpha_{k}\right|=\mathbf{D}_{i}^{H}[k]\left|\mathbf{r}_{i, j}\right| /\left\|\mathbf{D}_{i}[k]\right\|_{2}^{2} .
$$

The estimated $\widetilde{k}$ is the one which minimizes the residual, i.e.,

$$
\widetilde{k}=\arg \min _{1<k<M_{i}}|| \mathbf{r}_{i, j}|-| \alpha_{k} \mid \mathbf{D}_{i}[k] \|_{2}^{2},
$$

To be specific, if the difference between the assumed value and the real value is less than a minimum signal power, then the type of the node is determined.

Furthermore, a check procedure is proposed to determine if it is a single-ton, according to the following criterion

$$
\left\|\left|\mathbf{r}_{i, j}\right|-\left|\widetilde{\alpha}_{\widetilde{k}}\right| \mathbf{D}_{i}[\widetilde{k}]\right\|_{2}^{2} / M_{i} \leq\left(\delta_{\min }^{2} \varepsilon_{2}+\sigma_{\min }^{2}\right),
$$

where $\delta_{\min }^{2}$ denotes the minimum single power, $\sigma_{\min }^{2}$ is the minimum noise power, and $\varepsilon_{2}$ is the single-ton detection threshold.

Different from the pervious beam alignment algorithm [7], which cannot obtain the Doppler shift parameters of the channel, in our scheme, a ML method is utilized to estimate the CFO of a single-ton bin as follows:

$$
\widetilde{f_{l}^{c}}=\arg \max _{f_{k}}\left|\mathbf{F}^{H}\left(f_{k}\right) \mathbf{r}_{i, j}\right| /\left\|\mathbf{F}^{H}\left(f_{k}\right)\right\|_{2}^{2}\left\|\mathbf{r}_{i, j}\right\|_{2}^{2},
$$

where $\mathbf{F}(.) \in \mathbb{C}^{N \times 1}$ denotes the CFO steering vector, and $f_{k} \in$ $\left[0, f^{\max }\right]$ is the CFO of the candidate. Thus, the real complex gains can be given by

$$
\widetilde{\alpha}_{k}=\mathbf{F}^{H}\left(\widetilde{f_{l}^{c}}\right) \mathbf{r}_{i, j} /\left\|\mathbf{F}^{H}\left(\widetilde{f_{l}^{c}}\right)\right\|_{2}^{2}
$$

Subsequently, by utilizing the Peeling Off method, we can obtain all the non-zero entries of the virtual angular domain representation. In addition, we can use multiple iterations to improve the success rate of detection. Unlike traditional iteration-based messaging method [3], which relies algorithm convergence, our algorithm solves this problem by single hard decision. Finally, the detail steps of our proposed method are listed in Algorithm 1.

\section{Performance Analysis}

We now discuss the performance of our proposed scheme, including the computational complexity and the CRLB. Firstly, the computational complexity order of the proposed algorithm mainly arises from ML algorithm in (9) and (12). Thus, the complexity order of the proposed beam alignment algorithm is $\mathcal{O}\left(M_{\max } N+J N\right)$, where $M_{\max }$ denotes the maximum number of non-zeros entries in each row of the coding matrix $G$,
Algorithm 1 Beam Alignment Algorithm Using Sparse-Graph Codes

Input: Received signals $\mathbf{r}$, sparse coding matrix $\mathbf{G}$ and bin detection matrix $\mathbf{D}_{\mathbf{i}}$ for all stages, number of iterations $O$, preset CFO estimation accuracy $J$, error threshold $\varepsilon_{1}$, and error threshold $\varepsilon_{2}$

Output: The estimated index of beam channel $\widetilde{b}$ and CFO $\widetilde{f^{c}}$. for iteration $1<0<O$ do

for stage $1<i<d$ do

for bin $1<j<f_{i}$ do

if $\left|\mathbf{r}_{i, j}[1]\right|^{2} \leq\left(\delta_{\min }^{2} \varepsilon_{1}+\sigma_{\min }^{2}\right)$ then

$\mathbf{r}_{i, j}$ is a zero-ton bin vector.

else

for index $1<k<M_{i}$ do

get the estimated index-value pair $\left\langle\widetilde{k}_{k},\left|\widetilde{\alpha}_{\widetilde{k}}\right|\right\rangle$ by using (9), (10).

identify if the received bin vector is a singleton by using (11).

if single-ton = 'true' then

obtain $\widetilde{b}$ by utilizing the estimated $\widetilde{k}$ and the coding pattern in the $j$-th bin of the $i$-th stage.

get the estimated CFO $\widetilde{f_{l}^{c}}$ and estimated path gain $\widetilde{\alpha}_{\widetilde{k}}$ by using (12), (13).

rebuild the virtual time-varying representation $\widetilde{\mathbf{h}}[\widetilde{b}]$.

peeling off: $\mathbf{r}=\mathbf{r}-\boldsymbol{\psi} \widetilde{\mathbf{h}}[\widetilde{b}]$.

else

bin vector $\mathbf{r}_{i, j}$ is a multi-ton bin vector.

end if

end for

end if

end for

end for

end for

and $J$ denotes the preset CFO estimation accuracy. Compared with the recently proposed random search (RS) algorithm [5], [6], which has the complexity order $\mathcal{O}\left(N_{\mathrm{RF}} M_{\mathrm{RF}} \times M_{\mathrm{R}} N_{\mathrm{T}}\right)$, our proposed scheme achieves a lower complexity. Secondly, since the received time-varying data can be modeled as a three-dimensional tensor $\gamma \in \mathbb{C}^{N_{R F} \times P \times P}$ for each successful bin detection, the CRLB results for the channel parameters $\left\{a_{l}, \theta_{l}, \varphi_{l}, f_{l}^{c}\right\}$ are derived in Appendix $\mathbf{B}$.

\section{Simulation Results}

In this section, we evaluate the performance of the proposed beam alignment scheme through numerical simulations. Here, we consider a mmWave Massive MIMO sytem with hybrid precoding architecture at both the BS and UE, where $N_{\mathrm{T}}=$ $64, M_{\mathrm{R}}=16$, and both of them have multiple RF chains. In particular, for the mmWave time-varying channel parameters, we set $T_{s}=1 \mu \mathrm{s}$, and the CFO $f_{l}^{c}$ is randomly distributed in $\left[0, f_{\max }^{c}\right]$. For the proposed algorithm, we utilize a regular graph $\Re_{d}^{K}(F, m)$ to construct the measurement matrix, $d \geq$ 3 stages. Furthermore, to strike a good balance between the 


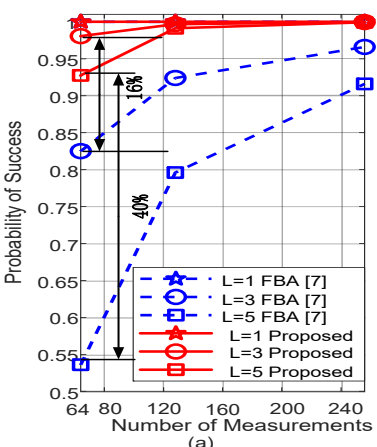

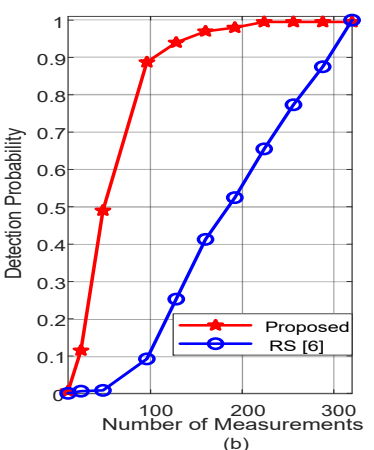

Fig. 4: Performance comparison of different algorithms in different cases. (a) noiseless case with $f_{\max }^{c}=0 \mathrm{~Hz}$, (b) noise case with $f_{\max }^{c}=10 \mathrm{kHz}$ and $\mathrm{SNR}=5 \mathrm{~dB}$.

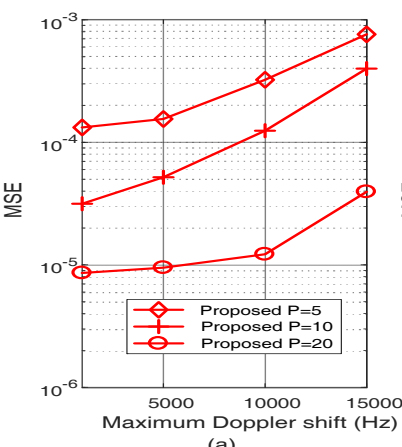

(a)

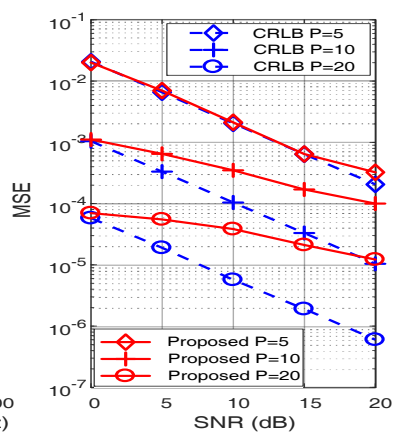

(b)
Fig. 5: MSE performance of the CFO estimation. (a) SNR = $20 \mathrm{~dB}$ vs. different maximum Doppler shifts, (b) $f_{\max }^{c}=10 \mathrm{kHz}$ vs. different SNRs.

performance and complexity order, we take $O=4$.

Fig. 4 compares the performance of our algorithm to that of the two existing beam alignment algorithms [6], [7] in terms of the probability of successful detection. As shown in Fig.4(a), compared with the fast beam alignment (FBA) algorithm [7] which is also based on the sparse coding, our algorithm provides considerable performance gains up to $16 \%$ $(L=3)$ and $40 \% \quad(L=5)$ even the number of measurements is very small (64). The benefit is due to the fact that the proposed algorithm takes advantage of the peeling off method and the density evolution to guarantee the bound achieving performance. Furthermore, in the noise case, our algorithm outperforms the RS algorithm [5], [6] v.s. different number of measurement. In particular, the detection probability denotes the probability of detecting the strongest path [6]. Although the performance of the random search method can be improved by sending multiple beams, it requires a longer sequence to maintain the orthogonality of multiple beams. Furthermore, combined with the results of computational complexity analysis in Section III-C, our algorithm achieves a lower computational complexity and better performance than the existing beam-alignment algorithms.

In Fig. 5, the MSE performances of the CFO estimation v.s. different parameters are investigated. As can be seen in Fig. 5 (a), our propose algorithm can effectively estimate the CFO even the maximum Doppler shift is very large. Fig. 5 (b) shows that the proposed CFO estimation method can approach the theoretical CRLB under the condition of each successful detection. However, this only happens when $\mathrm{P}$ is relatively small. The proposed algorithm exhibits a certain error floor when $\mathrm{P}$ increases. According to the conclusion in [12], the alternate least squares (ALS) method can reach the CRLB, but at the cost of higher computational complexity. Furthermore, if $\mathrm{P}$ is too long, the total pilot overhead of our algorithm will be very high. Therefore, the simulation results show that our algorithm strikes a good balance between performance and computational complexity.

\section{CONCLUSION}

This paper proposed a beam alignment algorithm using sparse codes for mmWave time-varying channel beam index acquisition and CFO estimation. Two CRLBs of the proposed scheme are derived as a benchmark to evaluate the performance of our algorithm. Simulation results show that the proposed beam alignment scheme outperforms the existing beam alignment algorithms and has potentials to approach the CRLBs. Our future work will focus on applying our proposed algorithm to holographic MIMO surfaces scene [14] and reconfigurable intelligent surfaces scene [15].

\section{APPENDIX A \\ PROOF OF THEOREM 1}

Define $Y$ as the total number of edges that are not decoded over a randomly graph from the ensemble $\Re^{K}(F, m)$, similar to the results in [10] and [9], we have

$$
\begin{aligned}
& \mathbb{E}(Y)<2 K d p_{i}, \\
& P(|Y-\mathbb{E}(Y)|>K d \varsigma)<e^{-\delta \varsigma^{2} K^{1 /(4 i+1)},}
\end{aligned}
$$

where $\varsigma$ is the arbitrary parameter, and $p_{i}$ is the probability of the event that an edge exists after the $i$-th peeling-off operation.

Furthermore, to derive the expression of $p_{i}$, we define the edge degree distribution in the ensemble as

$$
\begin{aligned}
& \lambda(\alpha)=\sum_{i=1}^{\infty} \lambda_{i} \alpha^{i-1}, \\
& \rho(\alpha)=\sum_{i=1}^{\infty} \rho_{i} \alpha^{i-1},
\end{aligned}
$$

where $\lambda_{i}$ and $\rho_{i}$ are the probability that the edge connected to the left (resp. right) node with degree $i$. For the proposed 'beams-and-detections procedure, $\lambda(\alpha)=\alpha^{d-1}$. Furthermore, considering that the degree of a right node follows the binomial distribution $B(1 / \eta K, K)$ [10], we have

$$
\begin{aligned}
\rho_{i} & =i \eta P(a \text { right node has edge degree } \mathrm{i}), \\
& \approx \frac{(1 / \eta)^{i-1} e^{-1 / \eta}}{(i-1) !} .
\end{aligned}
$$

Thus, the edge degree distribution polynomial $\rho(\alpha)$ is given by

$$
\rho(\alpha)=e^{(-(1-\alpha) / \eta)},
$$

Then, under the cycle-free assumption, the probability $p_{i}$ in (14) for the ensemble $\Re^{K}(F, m)$ can be expressed as

$$
p_{i+1}=\left(1-e^{-\frac{p_{i}}{\eta}}\right)^{d-1},
$$

where $p_{1}=1$, and the choice of $\eta$ is given to guarantee $p_{i+1}<$ $p_{i}$ e.g.., see Table I. 
According to (14), we can draw the conclusion that with high probability, the proposed peeling-off decoder captures all but an small fraction of the variable nodes. However, in a real application scenario, we need to ensure the recovery of all the variable nodes. Therefore, based on the above conclusions, we next study how to complete the decoding work process.

Without loss of generality, we consider a set of left nonzero nodes $P$ in the random graph $\Re^{K}(F, m)$, and the corresponding right neighborhood $N_{i}(P)$ of the $i$-th subset of the right nodes. Note that the proposed peeling-off decoder fails when there are no single-ton nodes in the $N_{i}(P)$ for $i=1, \cdots d$. Furthermore, a sufficient condition for the above hypothesis is that the average degree of all the nodes in the neighborhood of $P$ (i.e. $\left|N_{i}(P)\right|$ for all the i) is less than 2, such as $\left|N_{i}(P)\right|>|P| / 2$. Specifically, if the average degree of the right nodes is less than 2 , the single-ton must occur. Then, considering that $f_{i}=\eta K+O(1)$, we will discuss the probability of the opposite event $\Im$, i.e., $\max \left\{\left|N_{i}(P)\right|\right\}_{i=1}^{d} \leq|P| / 2$ as follows:

$$
\begin{aligned}
\operatorname{Pr}(\aleph) & <\prod_{i=1}^{d}\left(\frac{|P|}{2 f_{i}}\right)^{|P|}\left(\begin{array}{c}
f_{i} \\
|P| / 2
\end{array}\right) \\
& \approx\left(\frac{|P|}{2 \eta K}\right)\left(\begin{array}{c}
\eta K \\
|P| / 2
\end{array}\right)^{d} \\
& <\left(\frac{|P|}{2 \eta K}\right)\left(\frac{2 \eta K e}{|P|}\right)^{d|P| / 2} \\
& =\left(\frac{|P| e}{2 \eta K}\right)^{d|P| / 2}
\end{aligned}
$$

Furthermore, by utilizing a union bound, the probability of event $\aleph_{s}$ of some set of the variable nodes following the rules, i.e. $\max \left\{\left|N_{i}(P)\right|\right\}_{i=1}^{d} \leq|P| / 2$, we obtain

$$
\begin{aligned}
\operatorname{Pr}\left(\aleph_{s}\right) & <\operatorname{Pr}(\boldsymbol{\aleph})\left(\frac{K}{|P|}\right) \\
& <\left(\frac{|P| e}{2 \eta K}\right)^{d|P| / 2}\left(\frac{K e}{|P|}\right)^{|P|} \\
& <\left[\left(\frac{|P|}{\eta K}\right)^{d-2}\left(\frac{e}{2}\right)^{d}\left(\frac{e}{\eta}\right)^{2}\right]^{|P| / 2} \\
& \stackrel{(a)}{<} O\left((|P| / m)^{|P| / 2}\right)
\end{aligned}
$$

where $(a)$ comes from the fact that $d \geq 3$ and $m=O(\eta K)$. Then, according to $|P|=O(K)$, we obtain

$$
\operatorname{Pr}\left(\aleph_{s}\right)<O(1 / m),
$$

Finally, based on (14), (18) and (21), Theorem 1 can be proved.

\section{APPENDIX B}

\section{CRLB FOR MASSIVE-MIMO TIME-VARYING ChanNELS}

For each bin detection, the received data is utilized twice, once for amplitude information and once for complex information, it can thus be rewritten as

$$
\boldsymbol{\gamma}=\sum_{l=1}^{L} a_{l} \overrightarrow{\boldsymbol{\alpha}}_{\mathrm{BS}}\left(\theta_{l}\right) \circ \overrightarrow{\boldsymbol{\alpha}}_{\mathrm{MS}}\left(\varphi_{l}\right) \circ \boldsymbol{\beta}_{l}+\mathbf{N},
$$

where $\vec{\alpha}_{\mathrm{BS}}\left(\theta_{l}\right)=\mathbf{F}_{M S} \alpha_{\mathrm{BS}}\left(\theta_{l}\right), \vec{\alpha}_{\mathrm{MS}}\left(\varphi_{l}\right)=\mathbf{F}_{\mathrm{BS}} \alpha_{\mathrm{MS}}^{\dagger}\left(\varphi_{l}\right)$ $\beta_{l}=\left[e^{j 2 \pi f_{l}^{c}(s-1) P T_{s}} \cdots e^{j 2 \pi f_{l}^{c} s P T_{s}}\right]^{T}, s$ denotes the number of bin. Thus, all the unknown time-varying channel parameters $\left\{a_{l}, \theta_{l}, \varphi_{l}, f_{l}^{c}\right\}$ are contained in the vector $\gamma$. Consequently, similar to [12], [13], the CRLB of the channel parameter is given as follows:

$$
\begin{aligned}
\operatorname{CRLB}(\boldsymbol{\mu}) & =\Omega^{-1}(\boldsymbol{\mu}) \\
& =\mathbf{E}^{-1}\left\{\left(\frac{\partial f(\boldsymbol{\mu})}{\partial \boldsymbol{\mu}}\right)^{H}\left(\frac{\partial f(\boldsymbol{\mu})}{\partial \boldsymbol{\mu}}\right)\right\}
\end{aligned}
$$

where $\boldsymbol{\mu}=\left\{\boldsymbol{\alpha}^{T} \boldsymbol{\theta}^{T} \boldsymbol{\varphi}^{T}\left(\mathbf{f}^{c}\right)^{T}\right\}$ is the parameter vector, $\Omega(\boldsymbol{\mu})$ denotes the complex Fisher information matrix (FIM), and $f(\boldsymbol{\mu})$ is the log-likehood function.

\section{REFERENCES}

[1] K. Aldubaikhy, W. Wu, N. Zhang, N. Cheng and X. S. Shen, "mmWave IEEE 802.11ay for 5G Fixed Wireless Access," IEEE Wireless Communications, vol. 27, no. 2, pp. 88-95, April 2020.

[2] A. Alkhateeb, O. El Ayach, G. Leus and R. W. Heath, "Channel Estimation and Hybrid Precoding for Millimeter Wave Cellular Systems," IEEE Journal of Selected Topics in Signal Processing, vol. 8, no. 5, pp. 831-846, Oct. 2014.

[3] C. Huang, L. Liu, C. Yuen and S. Sun, "Iterative Channel Estimation Using LSE and Sparse Message Passing for MmWave MIMO Systems," IEEE Transactions on Signal Processing, vol. 67, no. 1, pp. 245-259, 1 Jan.1, 2019.

[4] C. Liu, M. Li, S. V. Hanly, P. Whiting and I. B. Collings, "MillimeterWave Small Cells: Base Station Discovery, Beam Alignment, and System Design Challenges," IEEE Wireless Communications, vol. 25, no. 4, pp. 40-46, August 2018.

[5] S. Chiu, N. Ronquillo and T. Javidi, "Active Learning and CSI Acquisition for mmWave Initial Alignment," IEEE Journal on Selected Areas in Communications, vol. 37, no. 11, pp. 2474-2489, Nov. 2019.

[6] X. Song, S. Haghighatshoar and G. Caire, "Efficient Beam Alignment for Millimeter Wave Single-Carrier Systems With Hybrid MIMO Transceivers," IEEE Transactions on Wireless Communications, vol. 18, no. 3, pp. 1518-1533, March 2019

[7] X. Li, J. Fang, H. Duan, Z. Chen and H. Li, "Fast Beam Alignment for Millimeter Wave Communications: A Sparse Encoding and Phaseless Decoding Approach," IEEE Transactions on Signal Processing, vol. 67, no. 17, pp. 4402-4417, 1 Sept.1, 2019.

[8] T. Okpotse and S. Yousefi, "Systematic Fountain Codes for Massive Storage Using the Truncated Poisson Distribution," IEEE Transactions on Communications, vol. 67, no. 2, pp. 943-954, Feb. 2019.

[9] T. J. Richardson and R. L. Urbanke, "The capacity of low-density paritycheck codes under message-passing decoding," IEEE Transactions on Information Theory, vol. 47, no. 2, pp. 599-618, Feb 2001.

[10] M. G. Luby, M. Mitzenmacher, M. A. Shokrollahi and D. A. Spielman, "Efficient erasure correcting codes," IEEE Transactions on Information Theory, vol. 47, no. 2, pp. 569-584, Feb. 2001.

[11] Q. Qin, G. Lin, P. Cheng, and B. Gong "Time-varying Channel Estimation for Millimeter Wave Multi-User MIMO Systems” IEEE Trans. Veh. Technol., vol. 67, no. 10, pp. 9435-9448, Oct. 2018.

[12] X. Liu and N. D. Sidiropoulos, "Cramér-Rao lower bounds for lowrank decomposition of multidimensional arrays," IEEE Trans. Signal Process., vol. 49, no. 9, pp. 2074-2086, Sep. 2001.

[13] Z. Zhou, J. Fang, L. Yang, H. Li, Z. Chen and R. S. Blum, "LowRank tensor decomposition-aided channel estimation for millimeter wave MIMO OFDM systems," IEEE J. Sel. Areas Commun., vol. 35, no. 7, pp. 1524-1538, Jul. 2017.

[14] C. Huang et al.,"Holographic MIMO Surfaces for 6G Wireless Networks: Opportunities, Challenges, and Trends," IEEE Wireless Communications, vol. 27, no. 5, pp. 118-125, October 2020.

[15] C. Huang, A. Zappone, G. C. Alexandropoulos, M. Debbah and C. Yuen, "Reconfigurable Intelligent Surfaces for Energy Efficiency in Wireless Communication," IEEE Transactions on Wireless Communications, vol. 18, no. 8, pp. 4157-4170, Aug. 2019. 